\title{
Additively manufactured metastructure design for broadband radar absorption
}

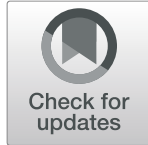

M. B. Abdullahi ${ }^{1,2^{*}}$ (iD and M. H. Ali ${ }^{1}$

\begin{abstract}
Background: Recent advances in material science and electronics led to the rapid development of communication devices and radar detection techniques resulting in an ever-increasing demand for improved stealth performance of air vehicles during scouts. Absorber design employing metastructure concept has recently become a popular approach to improving radar stealth performance. Metastructure permits the realization of desired absorption characteristics by careful design of geometrical structures and material compositions. In this study, a metastructure designed based on graphite SLS composite for radar absorption has been demonstrated. The unit cell of the proposed structure is simulated by COMSOL Multiphysics to determine the frequency-dependent absorption characteristic of the structure. It is fabricated by using a low-cost selective laser sintering technique of additive manufacturing technology.

Results: The prototype, while measured, shows effective absorption bandwidth of $1.04 \mathrm{GHz}$ that is in reasonable agreement with the simulated response of $2.08 \mathrm{GHz}$. The optimized structure exhibits $\leq-10 \mathrm{~dB}$ reflectivity within a broad frequency range extending from $7.60 \mathrm{GHz}$ to $18.00 \mathrm{GHz}$ under normal incidence in both TE and TM polarizations. Furthermore, the absorption performance under different polarizations and incident angles has been investigated. Results indicate that the absorber displays polarization indifference and exhibits a wide-angle of incidence tolerance of up to $45^{\circ}$ in TE polarization and $30^{\circ}$ in TM polarizations.

Conclusion: In this paper, the feasibility of using graphite SLS material to design and 3D print a metastructure design for radar absorbing has been established as confirmed by the simulation and the measurement results. The advantages of low cost, ultra-broad operating band, wide-angle of incidence feature, and polarization insensitivity qualifies the proposed absorber for stealth and electromagnetic shielding applications.
\end{abstract}

Keywords: Broadband, Graphite SLS, Metastructure, Radar, Wide-angle

\section{Background}

Recent advances in material science and electronics have led to the rapid development of communication devices and radar detection techniques. This has compounded the electromagnetic (EM) interferences and radiation problems. Also, there is pressing demand for improved stealth performance of air vehicles during the scout. In an attempt to find a solution to these problems, there has been extensive research interest in EM absorbing

\footnotetext{
* Correspondence: abdullahi.bello@udusok.edu.ng

'Department of Physics, Bayero University, Kano, Nigeria

${ }^{2}$ Department of Physics, Usmanu Danfodiyo University Sokoto, Sokoto, Nigeria
}

materials in the last few years. Current researches are aimed at developing EM absorbers with the properties of thinner thickness, lower density, wider angle of incidence tolerance, better polarization insensitivity, and optimal property mix that is peculiar to the area of application such as radar stealth [2-4], electromagnetic compatibility [5], energy harvesting [6], and so on. Recently, absorber design employing metastructure concept has become a popular approach [7-9] for improving radar stealth performance as it permits realization of 
desired absorption characteristics by carefully manipulating the geometry of the structure and/or material composition. Metastructures (MS) are artificially engineered subwavelength units that can effectively manipulate the propagation of electromagnetic waves resulting in unique electromagnetic features that are unattainable in conventional materials, such as near-zero or negative refractive index.

Additive manufacturing technology, which is also known as 3D printing, is a promising processing technology for fabricating structures and devices with different geometries using computer-aided design [10]. It offers high efficiency, convenience, and a low-cost fabrication process that involves printing successive layers of a given material on top of each other. Lately, 3D printing systems have been utilized to manufacture metamaterial absorbers (MMAs) of different structural designs and material bases [11-17]. However, the main hindrance for the fabrication of broadband MMAs using 3D printing technology is the limited range of materials compatible with $3 \mathrm{D}$ printers $[18,19]$. Most of the available 3D printing materials are fully insulating or have low conductivity values [11], which limits their usage in 3D printing of EM absorbing metamaterials. To enhance the dielectric performance of the common 3D printing polymers, organic materials such as carbon, carbon black, carbon nanotubes, and graphite are loaded to the polymers as hitherto demonstrated in non-3D printing polymeric composites [20, 21]. Nowadays, commercially available conductive filaments like conductive PLA, conductive ABS, and graphene are being explored for the development of EM absorbers and devices using 3D printing $[11-19,22]$. However, none of the existing reports reviewed have considered graphite SLS 3D printing material.

Therefore, a cross-block patterned broadband metastructure absorber for radar absorption at $\mathrm{X}$ and $\mathrm{Ku}$ bands manufactured using low-cost 3D printing technology is presented in this work. The graphite SLS material is introduced in the design of the absorber for the first time. A preliminary designed structure is $3 \mathrm{D}$ printed via selective laser sintering (SLS) technique and its absorption performance measured afterward. Good agreement between measured and simulated results of the preliminary absorber is observed. By optimizing the dimensions of the cross-block patterned preliminary absorber resonator unit cell, an ultra-broad absorption band with a reflectivity of below $-10 \mathrm{~dB}$ (absorptivity of higher than $90 \%$ ) is realized over the range of 7.6 to $18 \mathrm{GHz}$ radar spectrum as indicated in the simulation results. Moreover, the angular acceptance of the absorber in transverse electric (TE) and transverse magnetic (TM) mode are investigated, likewise, absorber polarization dependency is simulated. Results indicated wide angles of incidence tolerance for both polarization modes and polarization insensitivity at normal incidence. These exceptional promising features of the present metastructure absorber design qualifies it to be used for different applications in radar frequencies, such as low-cost electromagnetic interference shielding, electromagnetic compatibility, and stealth technology.

\section{Theory}

The electromagnetic absorption behaviour of a structure can generally be determined by using Eq. (1) where the frequency-dependent parameters $A(\omega), R(\omega)$, and $T(\omega)$ represent the absorption, reflectance, and transmittance, respectively.

$$
A(\omega)=1-|\mathrm{R}(\omega)|-|\mathrm{T}(\omega)|=1-\left|S_{11}\right|^{2}-\left|S_{21}\right|^{2}
$$

where $R(\omega)=\left|S_{11}\right|^{2}$ and $T(\omega)=\left|S_{21}\right|^{2}$

Due to the ground plane in the designed structure, $T(\omega)$ can be neglected. Therefore, Eq. (1) reduces to Eq. (2).

$$
A(\omega)=1-|\mathrm{R}(\omega)|=1-\left|S_{11}\right|^{2}
$$

Using scattering parameter $\left(S_{11}\right)$ from the simulations, the absorptivity of the RAMS is calculated using Eq. (2).

The wave equation of the propagating electric field " $E$ " based on the Maxwell equation is given by Eq. (3).

$$
\nabla \times \mu_{r}^{-1}(\nabla \times \boldsymbol{E})-k_{0}^{2}\left(\varepsilon_{r}-\frac{j \sigma}{\omega \varepsilon_{0}}\right) \boldsymbol{E}=0
$$

where $\mu_{r}$ represents the relative permeability, $k_{0}$ the free space wavenumber, $\varepsilon_{r}$ the relative permittivity, $\sigma$ the conductivity, $\omega$ the angular frequency, and $\varepsilon_{0}$ the permittivity of free space.

In COMSOL, the S-parameters describing the reflection and transmission of the wave are defined as follows:

$$
\begin{aligned}
S_{11} & =\frac{\int_{\partial \Omega}\left(E-E_{1}\right) \cdot E_{1}}{\int_{\partial \Omega} E_{1} \cdot E_{1}} \\
S_{21} & =\frac{\int_{\partial \Omega} E \cdot E_{2}}{\int_{\partial \Omega} E_{2} \cdot E_{2}}
\end{aligned}
$$

Then, the $S_{11}$ parameter evaluated from Eq. (4) is subsequently substituted into Eq. (2) in COMSOL software to calculate the magnitude of the absorptivity of the incident wave. Based on transmission line theory, impedance encountered by EM waves at layer " $\mathrm{i}$ " is defined as [23]:

$$
Z_{i n^{i}}=Z_{i} \frac{Z_{\text {in }}(i+1)+Z_{i} \tanh \gamma_{i} d_{i}}{Z_{i}+Z_{\text {in }}(i+1) \tanh \gamma_{i} d_{i}}
$$

where $Z_{i}$ and $\gamma_{i}$ can be represented as $Z_{i}=\sqrt{\frac{\mu_{r, i}}{\varepsilon_{r, i}}}$ and $\gamma_{i}=j 2 \pi f \frac{\sqrt{\varepsilon_{r}, \mu_{r, i}}}{C}$, respectively.

The magnitude of EM wave absorption represented by the reflectivity of an absorber backed by a perfect 


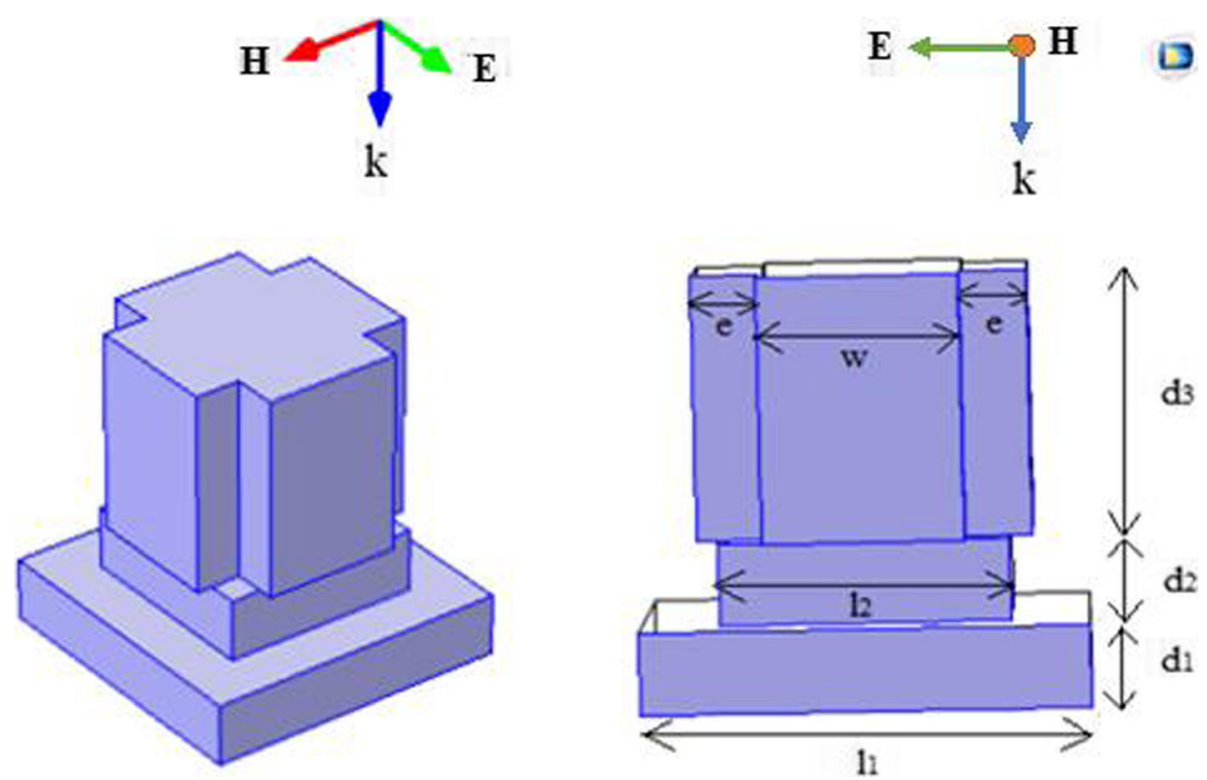

Fig. 1 Designed unit cell radar-absorbing metamaterial with three-layer cross-block structure. a Model of periodic unit. b Model size. $I_{1}=4.0 \mathrm{~mm}$, $I_{2}=2.6 \mathrm{~mm}, w=1.8 \mathrm{~mm}, e=0.6 \mathrm{~mm}, d_{1}=0.8 \mathrm{~mm}, d_{2}=0.8 \mathrm{~mm}, d_{3}=2.6 \mathrm{~mm}$

electrical conductor (PEC) ground plate can be written as Eq. (7).

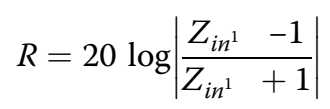

where $R$ is reflectivity and $Z_{i n^{1}}$ is surface layer input impedance.

The reflectivity of the three-layered RAMS can be calculated from Eqs. (6) and (7) using the FEM formulation.

\section{Method}

\subsection{Design of the structure}

The proposed absorber is a three-layer structure consisting of a cross-shaped surface structure, square block middle layer, and conventional single slab bottom layer as revealed in Fig. 1. The material used in the design of the absorber layers is Graphite SLS, exclusive material from a UK-based Graphite Additive Manufacturing company. It was obtained from the mixture of a Nylon 12 (PA) powder and graphite powder, to enhance the dielectric performance of the material. As graphite SLS assumed the property of graphite, the measured complex

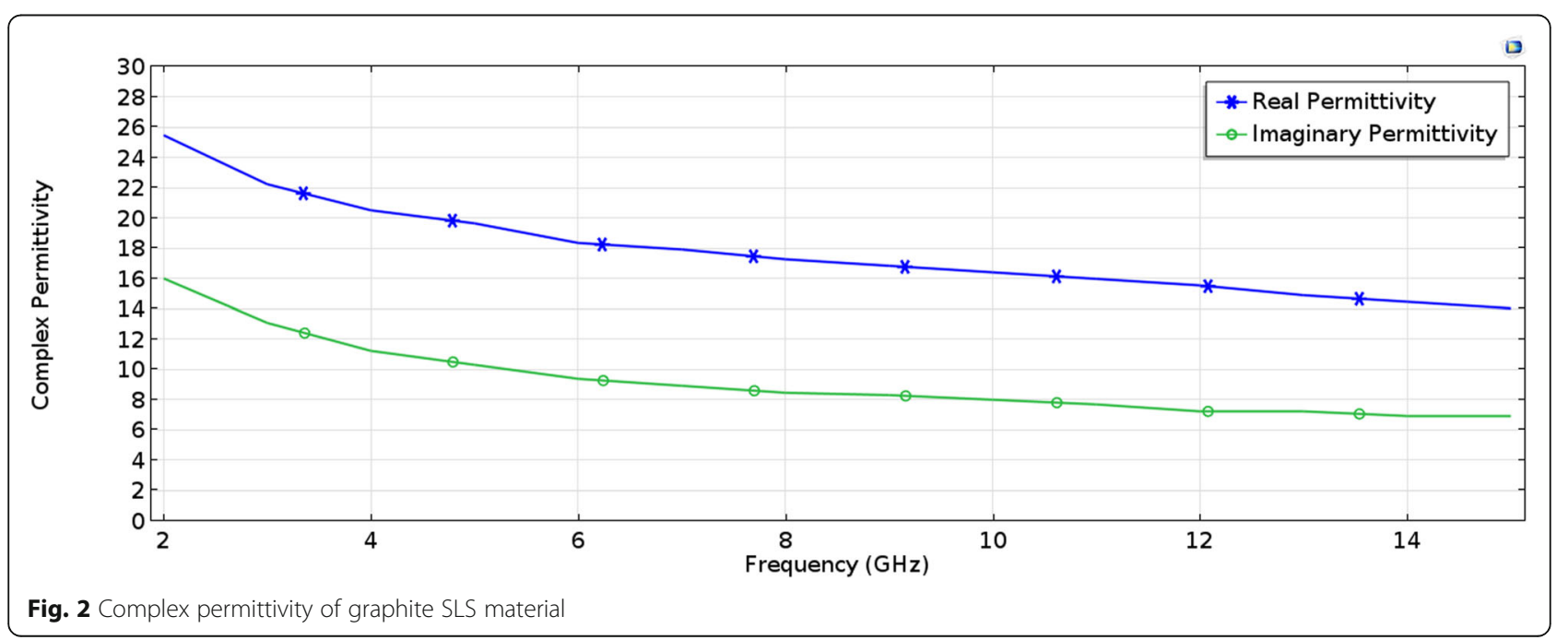




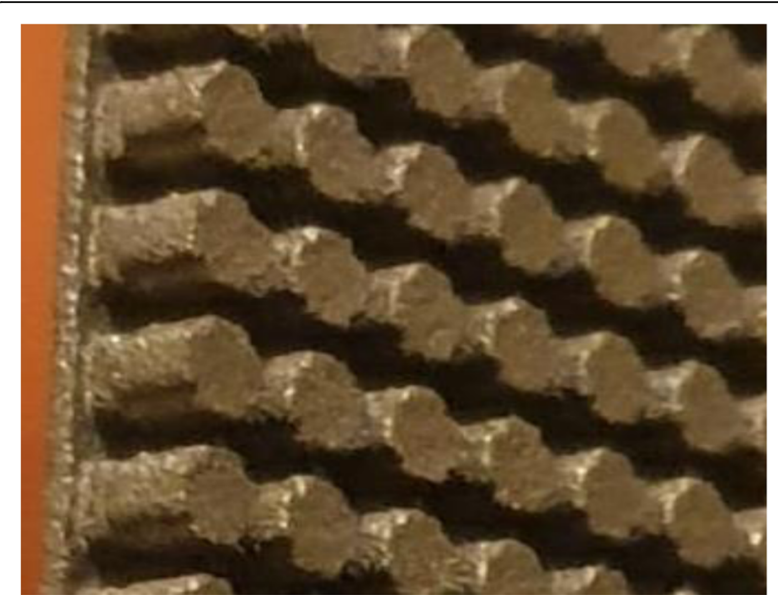

Fig. 3 Additively manufactured radar-absorbing metastructure

permittivity of graphite [24] used in this work is shown in Fig. 2, while the loss tangent of the graphite is 0.52. A copper film of $0.04 \mathrm{~mm}$ thickness is used in the design as a ground plane to prevent transmission of the waves beyond the structure. The schematic of the unit cell of the proposed radar-absorbing metastructure (RAMS) shown in Fig. 1 is simulated by using the frequency domain solver of COMSOL Multiphysics environment based on finite element method (FEM) to investigate its absorption characteristics.

\subsection{Simulation approach}

FEM is used in this study to investigate the EM absorbing property of the proposed design. In FEM, the simulation domain is discretized into sub-elements called mesh elements. Tetrahedral mesh element is selected with a maximum element size of one-tenth of the minimum wavelength $\left(\frac{1}{10} \lambda_{\min }\right)$ of the input wave to obtain reasonable accuracy of the simulation results within a reasonable computation resource in the COMSOL. The complete mesh consists of 16688 domain elements, 2294 boundary elements, and 276 edge elements. The boundaries of the simulation domain along $x$ and $y$ axes were simulated as a perfect magnetic conductor (PMC) and perfect electric conductor (PEC) boundary conditions as shown in Fig. 1b. This method sets tangential fields to zero [1] respectively to mimic an infinite periodic structure arranged in the $x$ and $y$ directions. A periodic port boundary in the radio frequency (RF) module of COMSOL was used to generate plane-polarized electromagnetic wave excitations along the $z$-axis plane. With this boundary condition, the magnitude of the reflection loss can be assessed. Impedance boundary condition (IBC) which treats any material behind the boundary as being infinitely large is chosen for the ground plane. It is used at boundaries where the field is known to penetrate only a short distance outside the boundary. This penetration is approximated by the IBC to avoid the need to include another domain in the model. The IBC is represented in Eq. (8).

$$
\sqrt{\frac{\mu_{0} \mu_{r}}{\varepsilon_{0} \varepsilon_{r}-\frac{j \sigma}{\omega}}} \mathbf{n} \times \mathbf{H}+\mathbf{E}-(\mathbf{n} . \mathbf{E}) \mathbf{n}=\left(\mathbf{n} . \mathbf{E}_{\mathrm{s}}\right) \mathbf{n}-\mathbf{E}_{\mathrm{s}}
$$

In Fig. 2, the measured dielectric properties of graphite SLS material [24] are shown. Together with the loss

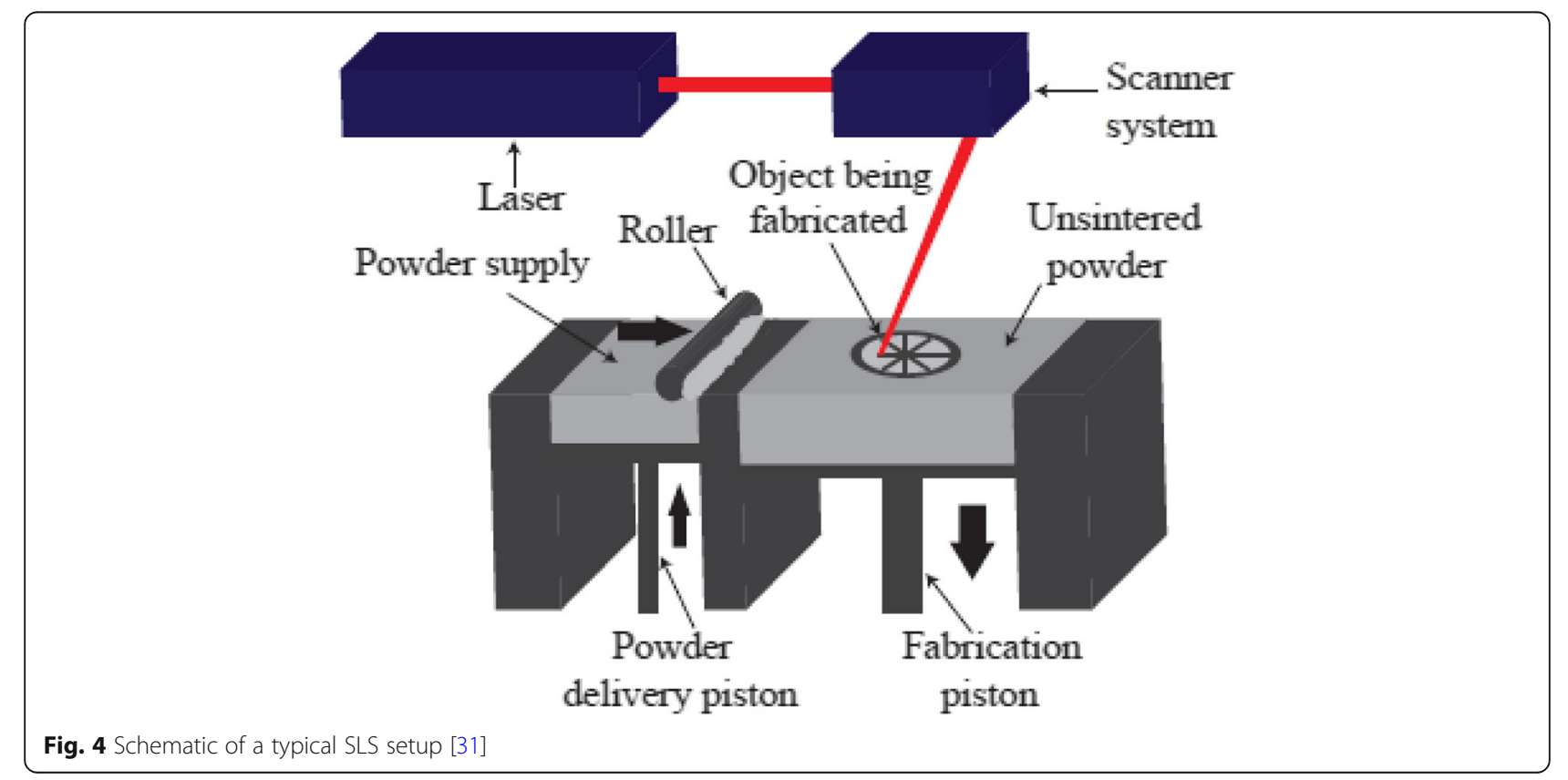




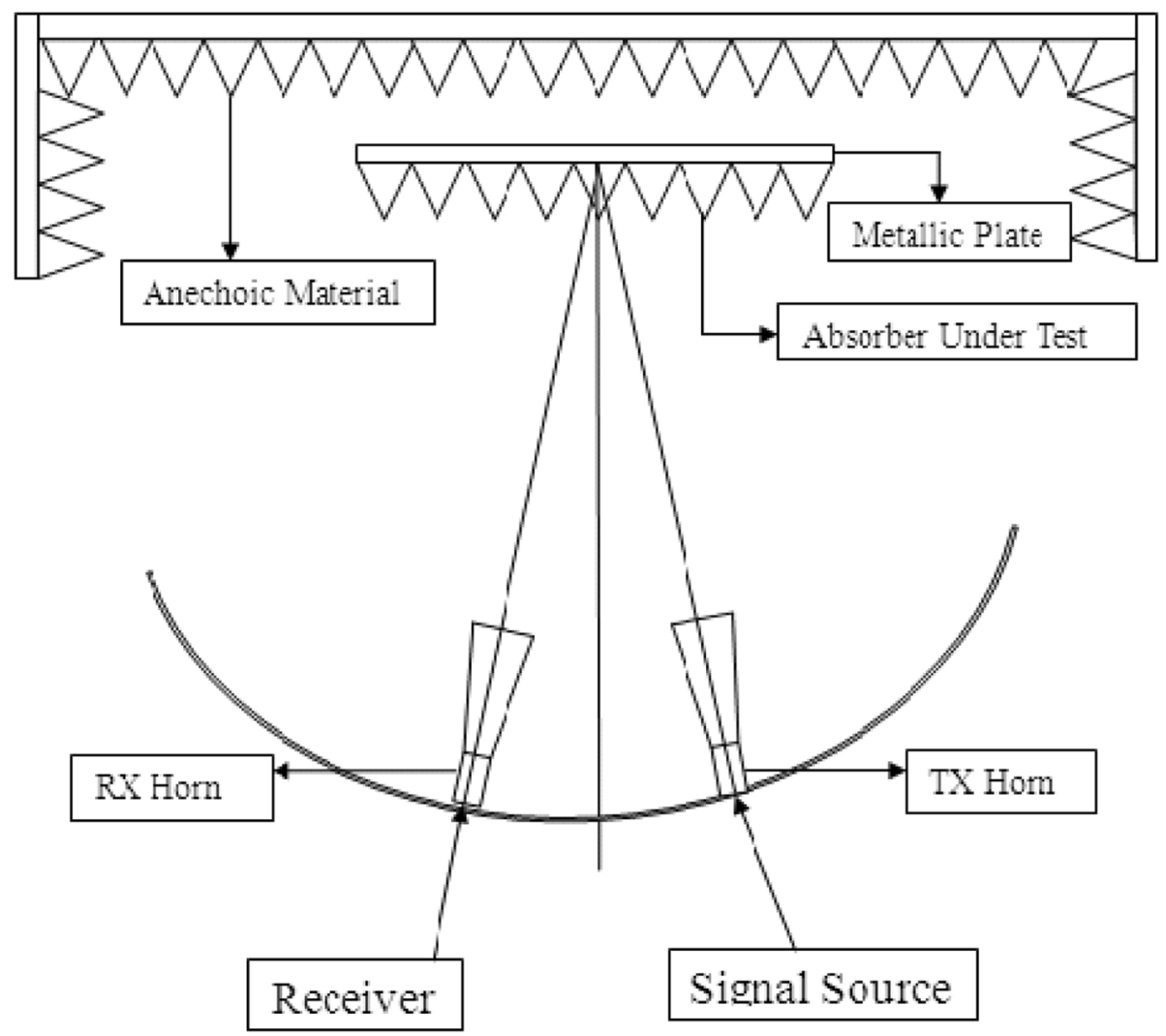

Fig. 5 Schematic of NRL arch method RL measurement setup

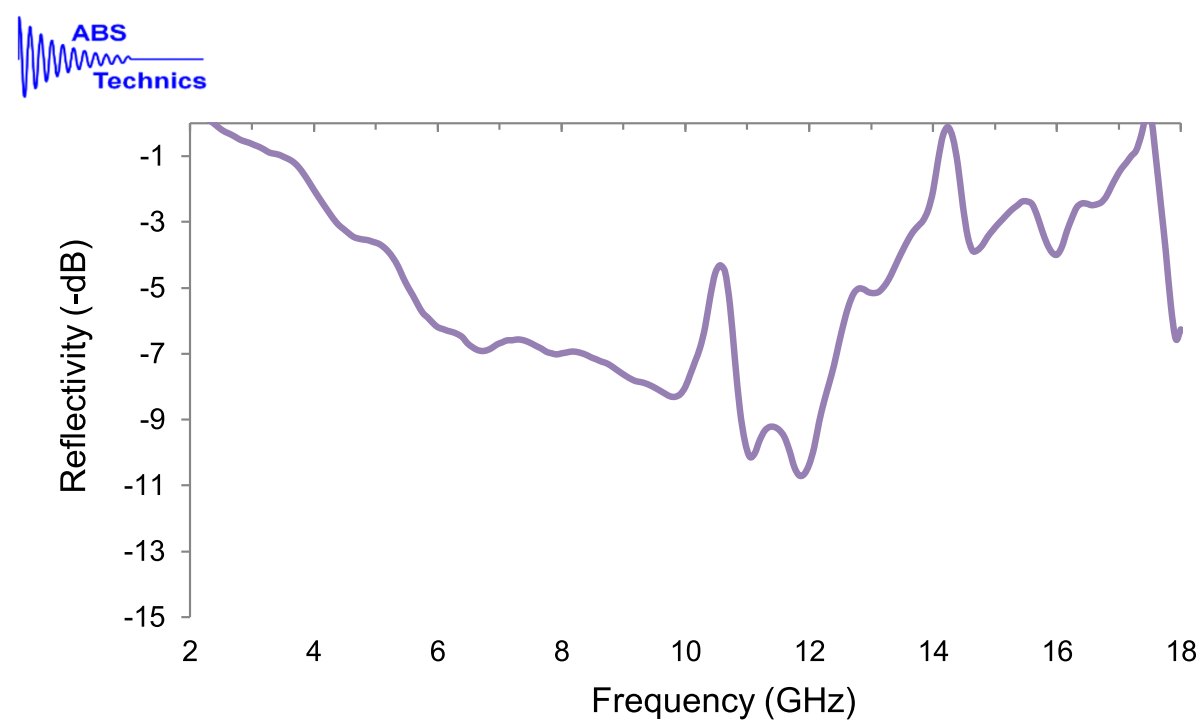

Fig. 6 Measured reflectivity plots at $10^{\circ}$ incidence of TE-polarized wave 


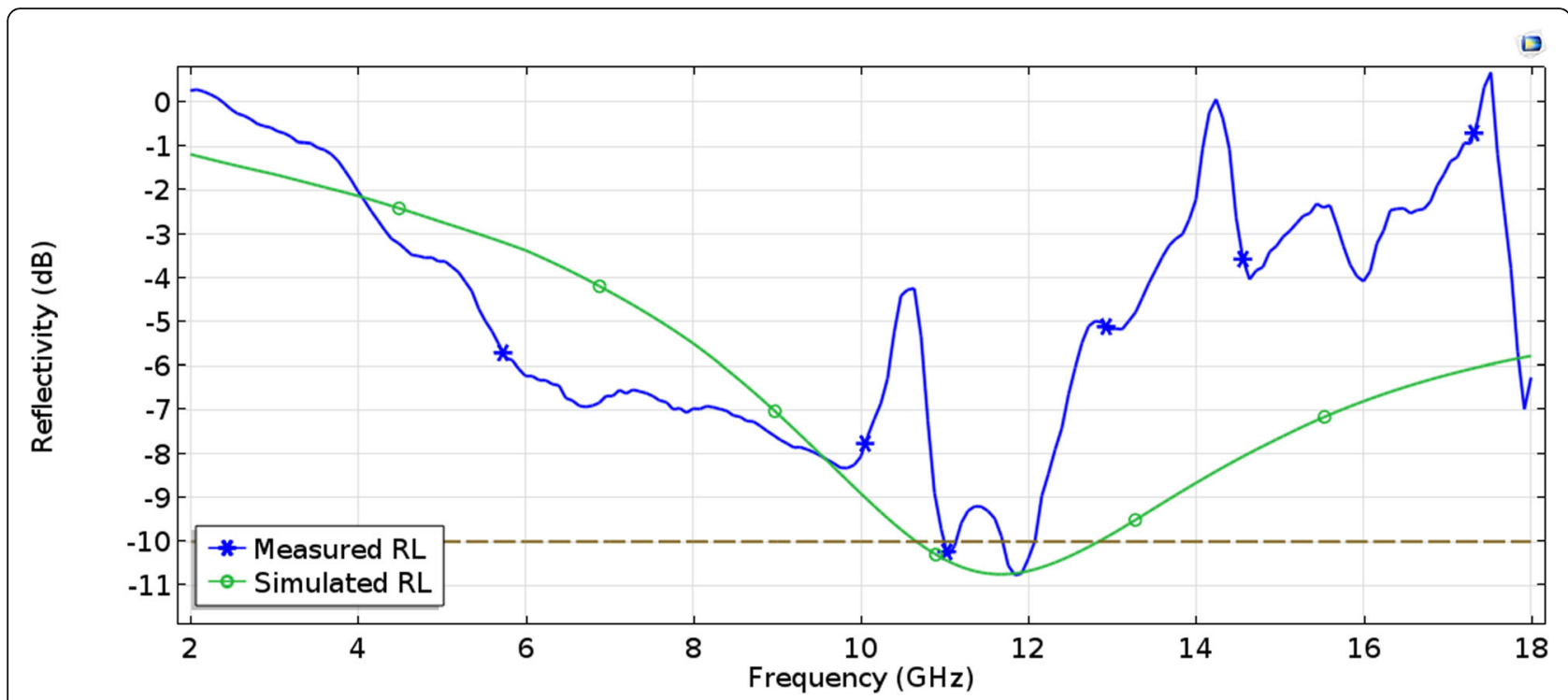

Fig. 7 Measured and simulated reflectivity plots at $10^{\circ}$ incidence of TE-polarized wave

tangent of material recorded as 0.52 , these material parameters are used in the simulation to evaluate the absorption behaviour of the proposed structure.

\subsection{Fabrication and measurement}

We used the services of UK based graphite additive manufacturing company for the fabrication of the proposed designed RAMS model. The RAMS was built using selective laser sintering (SLS) process and a partially enlarged picture of the 3D printed radar-absorbing metastructure is presented in Fig. 3.

Selective laser sintering (SLS) is an additive manufacturing process that allows the building of complex 3D parts by solidifying successive layers of powder material deposited on top of each other [25]. A wiper deposits a thin layer of powder particles (typically $0.1-0.3 \mathrm{~mm}$ thickness) on a fabrication bed, laser scans over the particles in the shape of the desired object as calculated from a CAD model causing the particles to melt and fuse. The final part is built by repeatedly depositing and sintering successive powder layers. A schematic overview of the SLS process is depicted in Fig. 4. No support structures are needed and allow rapid production of complicated geometries parts in one step process. Suitable materials for SLS include polymers, ceramics, and metals.

The fabricated absorber structure performance was measured at ABS technics [26] laboratory to verify the rationality of the Comsol Multiphysics design. NRL Arch measurement procedure was adopted to obtain the

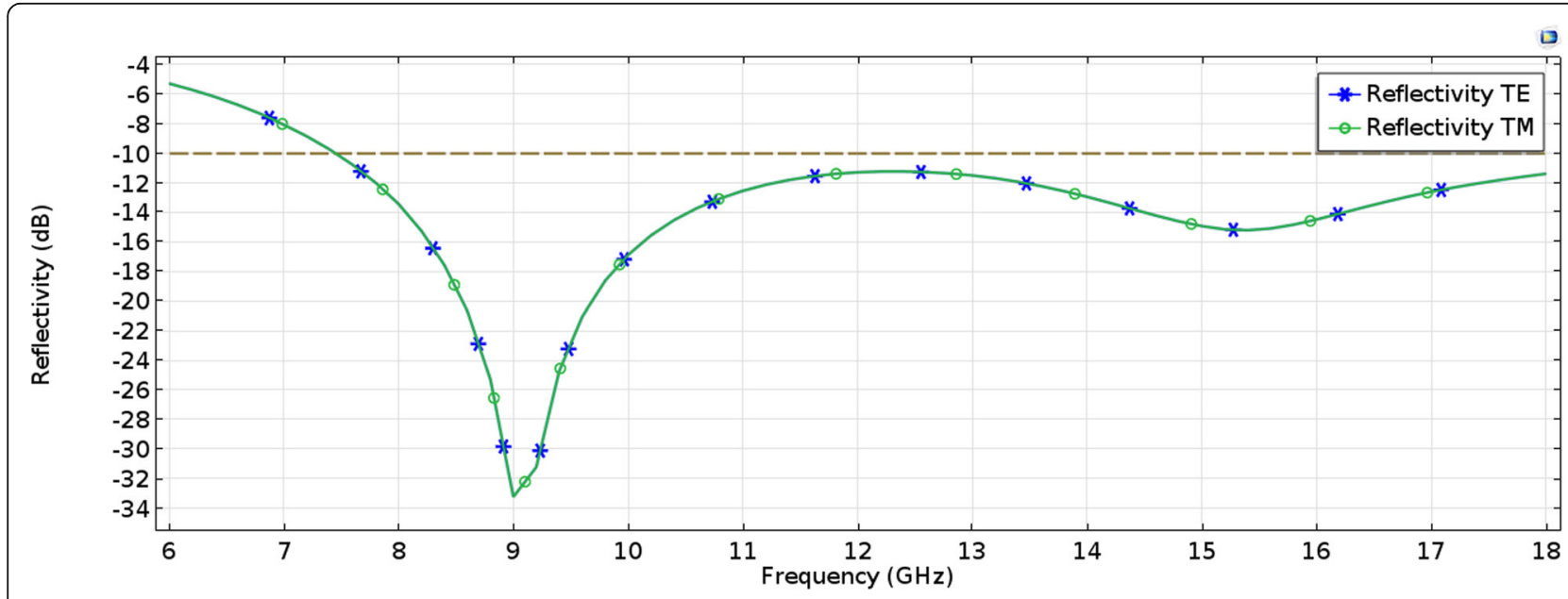

Fig. 8 Simulated reflectivity plots of the optimized structure at normal incidence for TE and TM polarizations 


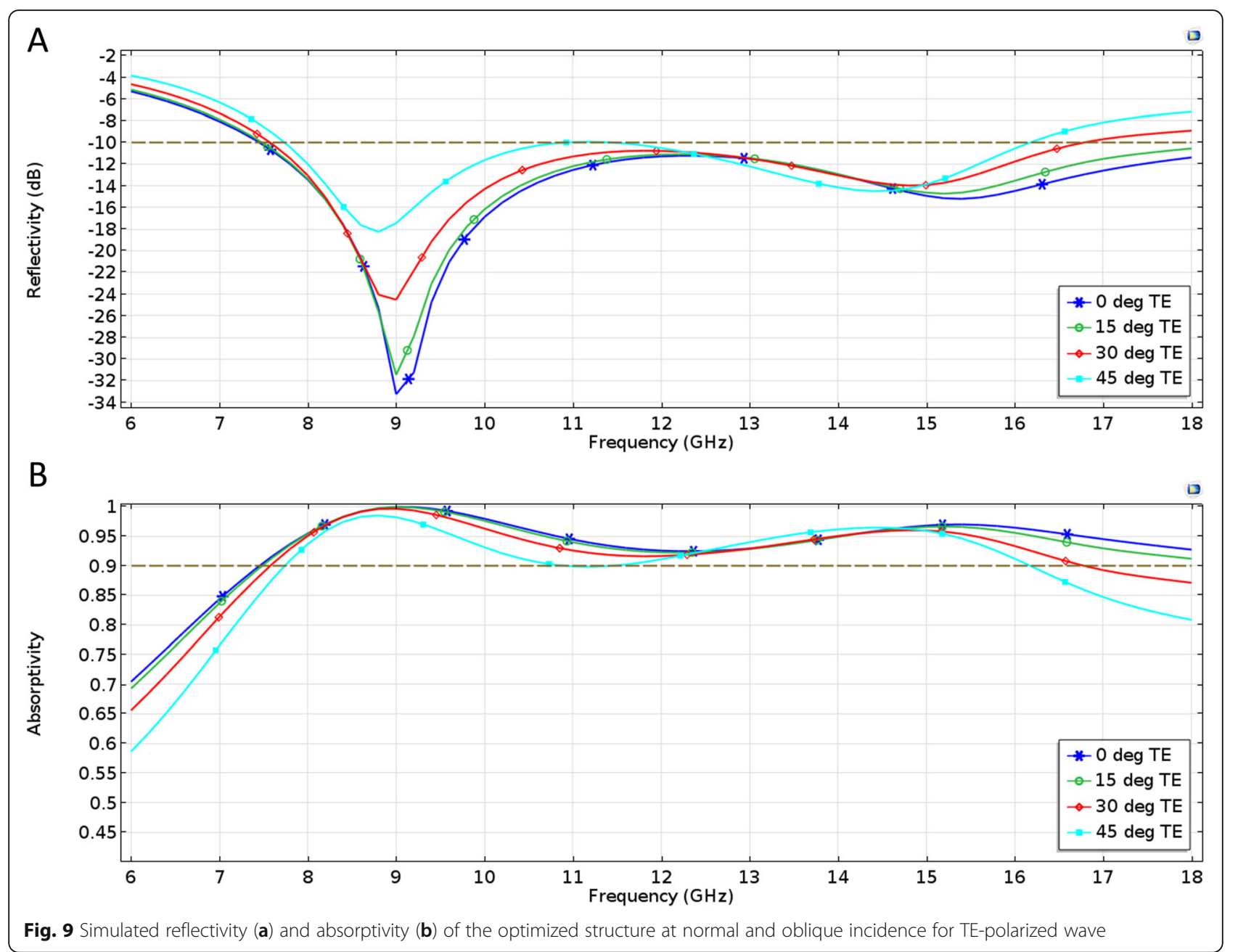

absorber reflection loss (RL). Measurement of RL at a near-normal incidence of $10^{\circ}$ for each antenna versus the normal on the reflective metal plate for TE polarized incident wave was carried out from $2.0-18 \mathrm{GHz}$. The schematic of the measurement setup is indicated in Fig. 5 and the detailed measurement method as per IEEE std 1128/ 1998 is given in [27].

\subsection{Geometric Dimension Optimization}

The measured and simulated results of the 3D printed RAMS demonstrate below $-10 \mathrm{~dB}$ reflectivity in the 10 $12 \mathrm{GHz}$ range only. To obtain broad absorption in the $\mathrm{X}$ and $\mathrm{Ku}$ bands of radar spectrum satisfying the below -10 $\mathrm{dB}$ reflectivity, the unit cell geometric dimensions are optimized by using Comsol Multiphysics' in-built Nelder-Mead method to get the desired absorption property.

\section{Results}

\subsection{Additively manufactured RAMS}

The measured reflectivity result of the $3 \mathrm{D}$ printed absorber at an incident angle of $10^{\circ}$ for TE-polarized wave is shown in Fig. 6, and the simulated reflectivity is plotted alongside the measured reflectivity in Fig. 7 for comparison.

\subsection{Geometric dimension optimized RAMS}

The optimized dimensions obtained which result in broadband absorption characteristics of the designed absorber are $l_{1}=15.0 \mathrm{~mm}, l_{2}=3.5 \mathrm{~mm}, w=4.2 \mathrm{~mm}, e=$ $1.6 \mathrm{~mm}, d_{1}=1.2 \mathrm{~mm}, d_{2}=0.6 \mathrm{~mm}, d_{3}=3.2 \mathrm{~mm}$. The simulated reflectivity of the optimized structure at normal incidence for both TE and TM polarizations is presented in Fig. 8.

Absorber angle of incidence tolerance is evaluated to assess its suitability for practical application which demands a wide angle of incidence performance. Simulations were carried out for TE and TM modes under different angles of incidence varying from $0^{\circ}$ to $45^{\circ}$ at steps of $15^{\circ}$. Absorber reflectivity and absorptivity responses under different angles of incidence for TE mode of the optimized design are displayed in Fig. 9, while absorber's angle of incidence responses for TM 

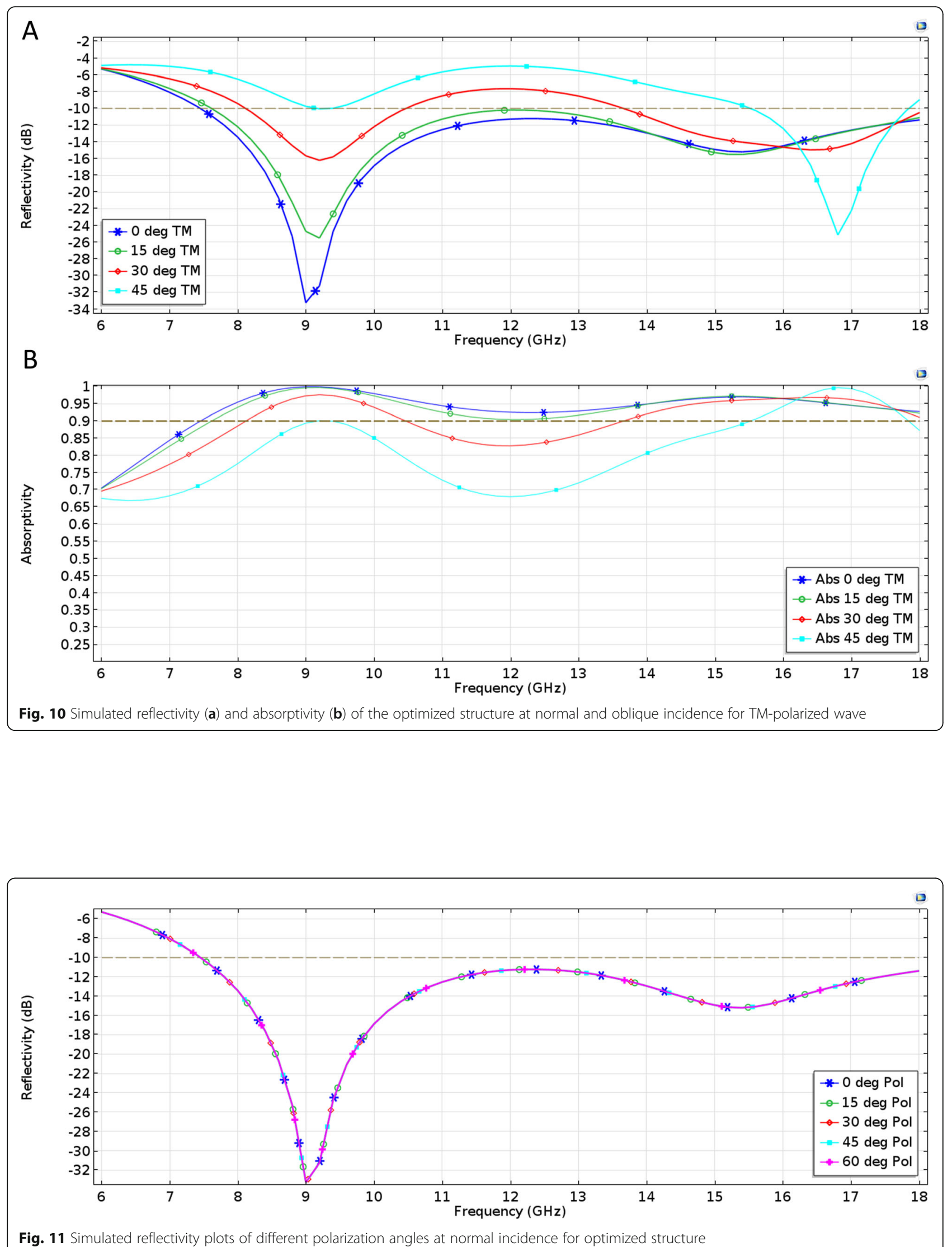

Fig. 11 Simulated reflectivity plots of different polarization angles at normal incidence for optimized structure 


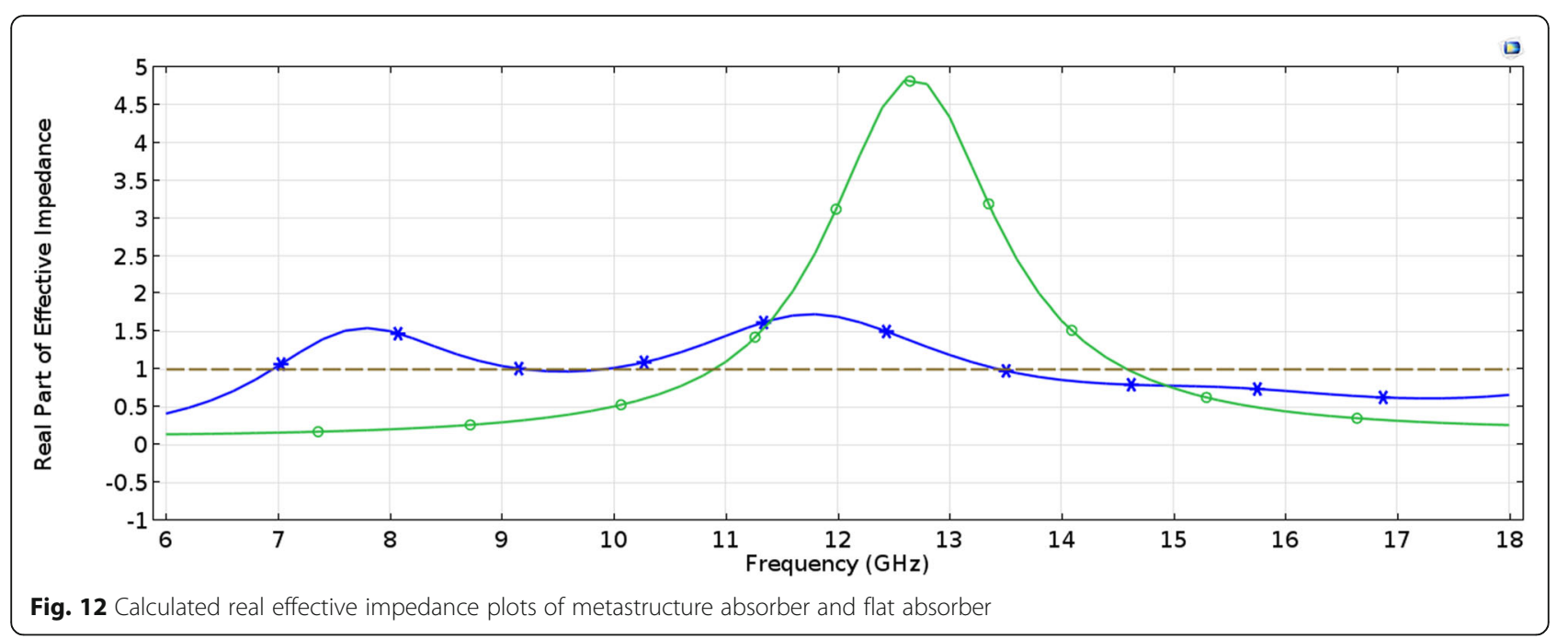

mode are depicted in Fig. 10. Another useful characteristic of an EM absorber is the ability of the absorber to respond well in different polarization angles. Therefore, the metastructure's response to different polarization angles is investigated in this paper and revealed in Fig. 11.

When the impedance of an absorber matches that of air, reflection is minimized which results in near-unity absorbance. For comparison, the real and imaginary parts of the impedance of the metastructure and single-layer flat designs were calculated from the simulated complex $S$ parameters using $Z_{\text {eff }}(\omega)=\sqrt{\frac{\left(1+S_{11}\right)^{2}}{\left(1-S_{11}\right)^{2}}}$ and plotted in Figs. 12 and 13. To explore the physical absorption mechanism of the proposed RAMS, current density, power loss density, and E field distributions were simulated at two absorption peaks of 9.0 and
$15.4 \mathrm{GHz}$ when the incident angle is $0^{\circ}$, and results are revealed in Fig. 14.

\section{Discussions}

Considering the additively manufactured RAMS, there is a favourable agreement between the simulation and measurement curves. The effective absorption bandwidth is $1.04 \mathrm{GHz}$ for measured and $2.08 \mathrm{GHz}$ for simulated. Effective absorption bandwidth is often defined as the corresponding frequency range in which reflectivity is $\leq-10 \mathrm{~dB}$. Deviations between simulated and measured results are observed, which can be attributed to the imperfections resulting from the fabrication and measurement processes.

The geometric dimension optimization has greatly improved the effective absorption bandwidth of the RAMS,

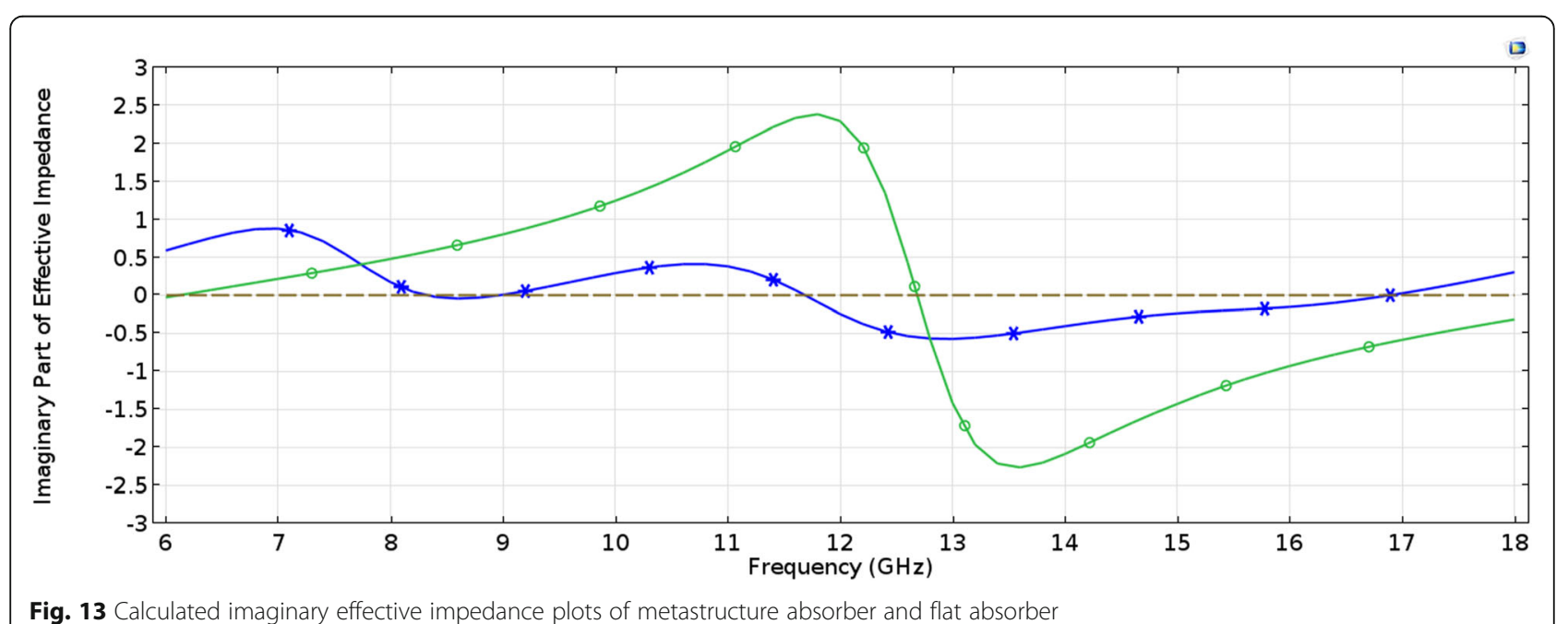

Fig. 13 Calculated imaginary effective impedance plots of metastructure absorber and flat absorber 


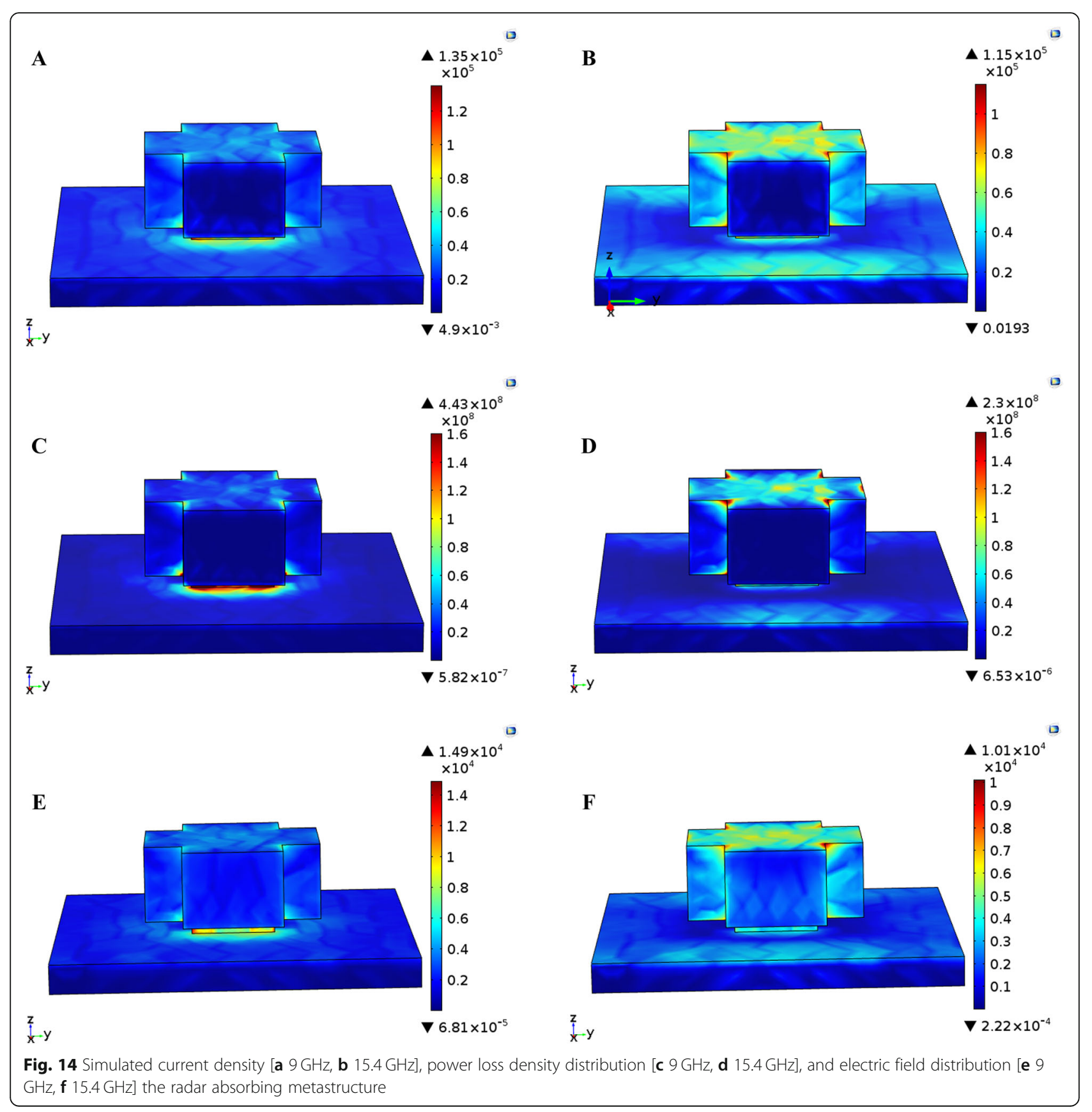

covering almost the entire frequency range of interest $(\mathrm{X}$ and Ku bands) as shown in Fig. 8. The new optimized absorber thickness is $5.0 \mathrm{~mm}$ only as against the $4.2 \mathrm{~mm}$ of the printed absorber. The reflection coefficient curves for the TE and TM polarizations are observed to overlapped at normal incidence. Moreover, it can be seen from Fig. 9a that as the angle of incidence increases to $15^{\circ}$, the effective bandwidth is unchanged which slightly decreases as the incident angle increase to $45^{\circ}$. Absorption levels are observed to diminish as the incident angle increases as depicted in Fig. 9b. For TM polarization, the effective bandwidth is similarly unchanged as the angle of incidence increases to $15^{\circ}$ while it decreases as the incident angle is varied to $45^{\circ}$ according to results from Fig. 10a. Absorption levels are observed to be greater than $90 \%$ for normal and $15^{\circ}$ incidences, greater than $80 \%$ at $30^{\circ}$, and greater than $70 \%$ at $45^{\circ}$ when TM wave interacts with the RAMS as depicted in Fig. 10b. It is, however, worth mentioning that the absorption level is greater than $70 \%$ in the $\mathrm{X}$ and $\mathrm{Ku}$ bands for all the incident angles considered 
Table 1 Comparison of - $10 \mathrm{~dB}$ absorption bandwidth range between radar-absorbing metastructure of this work and previous works

\begin{tabular}{lllll}
\hline Reference & Material & Frequency range $(\mathrm{GHz})$ & Effective absorption bandwidth & Thickness $(\mathbf{m m})$ \\
\hline$[13]$ & Conductive ABS & $4-12$ & 5.43 & 3.50 \\
{$[17]$} & Graphene/nano-Fe $\mathrm{O}_{4} / \mathrm{PLA}$ & $12.4-16.0$ & 3.60 & 3.00 \\
{$[18]$} & Conductive ABS & $3.9-12.0$ & 8.10 & 9.37 \\
{$[19]$} & Conductive PLA & $6.0-8.50$ & 2.50 & 5.04 \\
This work & Graphite SLS & $7.6-18.0$ & 10.40 & 5.00 \\
\hline
\end{tabular}

either for TE wave or TM wave while the absorption level is said to generally decrease with the increase of angle of incidence.

Simulation results for polarization angles varying from $0^{\circ}$ to $60^{\circ}$ indicated polarization independence behaviour as shown in Fig. 11. The curves are observed to overlapped over one another which is attributed to the fourfold symmetry of the structural design of the absorber unit cell. It is a fact that structures exhibiting fourfold symmetry demonstrate polarization insensitivity absorption behaviour [28-30]. Concluding, the designed RAMS is said to exhibit a wide-angle of incidence property and polarization-insensitive behaviour.

It can be spotted from Figs. 12 and 13 that the designed metastructure's effective impedance is much closer to matching air impedance than the singlelayer flat design, though both are designed from the same material and thickness. The real part impedance of the metastructure design from Fig. 12 is close to unity in most of the absorber working frequencies. From Fig. 13, the imaginary part of the effective impedance is observed to be near zero in most of the absorber's working frequency bands. This illustrates the effectiveness of the novel metastructure design for broadband radar spectrum absorption.

As depicted in Fig. 14a, the current density at the 9.0 $\mathrm{GHz}$ absorption peak is concentrated at the middle layer and topmost part of the surface layer. At $15.4 \mathrm{GHz}$, the current density distribution is dominant at the cross-shaped surface layer and the surface of the bottom layer according to Fig. 14b. Remarkably, the power loss distribution coincides well with the current density distribution as revealed in Fig. 14c, d, indicating that the current density plays a vital role in radar absorption which is mainly caused by ohmic losses. The electric field distribution shown in Fig. 14e, $\mathrm{f}$ is similar to that of the current density and power loss distribution.

Table 1 is a comparison of the designed absorber in this paper with some other absorbers fabricated by 3D printing. Compared with other absorbers in the literature listed in Table 1, the RAMS reported in this paper has a wider effective bandwidth at $10.40 \mathrm{GHz}$. It also yields a good thickness at $5.0 \mathrm{~mm}$, better than the absorber thickness obtained in [18, 19]. Moreover, Graphite SLS was for the first time introduced in this paper as can be observed from Table 1.

Finally, the present metastructure design can be described as a low-cost polarization-independent radar absorber with a wide-angle of incidence characteristics for $\mathrm{X}$ and $\mathrm{Ku}$ bands applications.

\section{Conclusion}

In this paper, we demonstrated the feasibility of using graphite SLS material to design and fabricate a metastructure for radar absorption using 3D printing as confirmed by the simulation and measurement results. The simulation results obtained from the optimized RAMS show that the structure can realize the below $-10 \mathrm{~dB}$ reflectivity in the 7.6-18.0 GHz frequency band at normal incidence of waves for TE and TM waves. This property is maintained at an oblique incidence until $45^{\circ}$ in TE wave and $15^{\circ}$ in the TM wave. However, the reflectivity is below $-5 \mathrm{~dB}$ (more than is $70 \%$ absorptivity) at $45^{\circ}$ incidences for TM wave. The metastructure absorber contributes to impedance matching to air that ensures minimized reflection and subsequent broadband absorption. Moreover, the proposed RAMS demonstrate polarization insensitivity and cost-effectivity as it is made from commercially available $3 \mathrm{D}$ printing material and technology thereby making it to be a practically viable candidate for stealth and electromagnetic interference shielding applications.

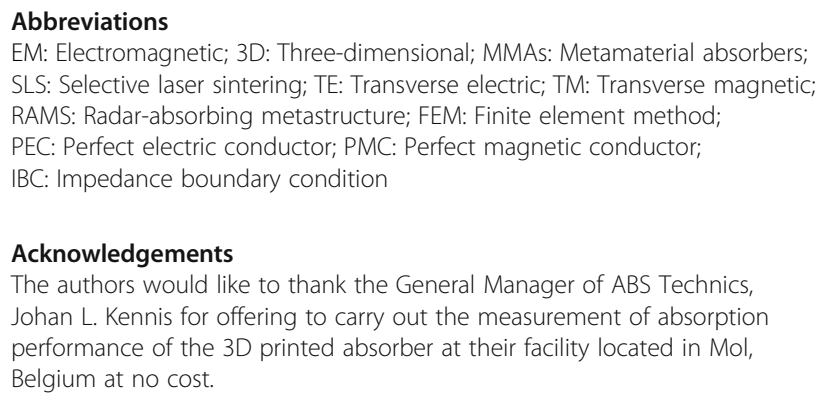

Acknowledgements

The authors would like to thank the General Manager of ABS Technics, Johan L. Kennis for offering to carry out the measurement of absorption performance of the 3D printed absorber at their facility located in $\mathrm{Mol}$, Belgium at no cost.

Authors' contributions

M.H. Ali conceived the idea, gives directives, suggested the fabrication method, and corrected the manuscript. M.B. Abdullahi carried out the design 
and the simulation and drafted and organized the manuscript. The authors have read and approved the final manuscript.

\section{Funding}

No funding received.

\section{Availability of data and materials}

Not applicable.

\section{Declarations}

Ethics approval and consent to participate

Not applicable.

\section{Consent for publication}

Not applicable.

\section{Competing interests}

The authors declare that they have no competing interests.

Received: 28 December 2020 Accepted: 22 March 2021

Published online: 09 April 2021

\section{References}

1. Luo H, Chen F, Wang X, Dai W, Xiong Y, Yang J, Gong R (2019) A novel two-layer honeycomb sandwich structure absorber with high- performance microwave absorption. Compos Part A 119:1-7. https://doi.org/10.1016/j. compositesa.2019.01.015

2. Zhang $L$ et al (2014) A broadband radar absorber based on perforated magnetic polymer composites embedded with FSS. IEEE Trans Magn 50(5): 4004305 pp $1-5$

3. Abdalla MA, Hu Z (2012) On the study of development of $x$ band metamaterial radar absorber. Adv Electromagn 1(3). https://doi.org/10.771 6/aem.v1i3.25

4. Xu H, Bie S, Xu Y, Yuan W, Chen Q, Jiang J (2016) Broad bandwidth of thin composite radar absorbing structures embedded with frequency selective surfaces. Compos Part A 80:111-117. https://doi.org/10.1016/j.compositesa.2 015.10.019

5. Chung DDL (2001) Electromagnetic interference shielding effectiveness of carbon materials. Carbon N Y 39(2):279-285. https://doi.org/10.1016/S00086223(00)00184-6

6. Hossain MJ, Faruque MRI, Islam MT (2018) Perfect metamaterial absorber with high fractional bandwidth for solar energy harvesting. PLoS One 13(11):1-15

7. Fan Q, Yang X, Lei H, Liu Y, Huang Y, Chen M (2020) Gradient nanocomposite with metastructure design for broadband radar absorption. Compos Part A Appl Sci Manuf 129:105698. https://doi.org/10.1016/j. compositesa.2019.105698

8. Huang Y, Yuan X, Chen M, Song WL, Chen J, Fan Q, Tang L, Fang D (2018) Ultrathin flexible carbon fiber reinforced hierarchical metastructure for broadband microwave absorption with nano lossy composite and multiscale optimization. ACS Appl Mater Interfaces 10(51):44731-44740. https://doi.org/10.1021/acsami.8b16938

9. Xie S, Zhu L, Zhang Y, Ji Z, Wang J (2020) Three-dimensional periodic structured absorber for broadband electromagnetic radiation absorption. Electron Mater Lett 16(4):340-346. https://doi.org/10.1007/s13391-020-00219-y

10. Nadgorny M, Ameli A (2018) Functional polymers and nanocomposites for $3 d$ printing of smart structures and devices. ACS Appl Mater Interfaces 10(21):17489-17507. https://doi.org/10.1021/acsami.8b01786

11. Tasolamprou AC, Mentzaki D, Viskadourakis Z, Economou EN, Kafesaki M Kenanakis G (2020) Flexible 3D printed conductive metamaterial units for electromagnetic applications in microwaves. Materials (Basel) 13(17):1-14. https://doi.org/10.3390/ma13173879

12. Jiang W, Yan L, Ma H, Fan Y, Wang J, Feng M, Qu S (2018) Electromagnetic wave absorption and compressive behavior of a three-dimensional metamaterial absorber based on 3D printed honeycomb. Sci Rep 8(1):1-7. https://doi.org/10.1038/s41598-018-23286-6

13. Lai W, Wang Y, He J (2020) Electromagnetic wave absorption properties of structural conductive ABS fabricated by fused deposition modeling. Polymers (Basel) 12(06):13. https://doi.org/10.3390/polym12061217
14. Lu Y, Chi B, Liu D, Gao S, Gao P, Huang Y, Yang J, Yin Z, Deng G (2018) Wideband metamaterial absorbers based on conductive plastic with additive manufacturing technology. ACS Omega 3(9):11144-11150. https://doi.org/10.1021/acsomega.8b01223

15. Kronberger R, Soboll P (2016) 3D-printed frequency selective surfaces for microwave absorbers. In: Proceedings of ISAP2016, Okinawa, Japan, pp 178179. https://doi.org/10.1109/MWSYM.2015.7166769

16. Zhou D, Huang X, Du Z (2017) Analysis and design of multi-layered broadband radar absorbing metamaterial using 3D printing technologybased method. IEEE Antennas Wirel Propag 16:133-136. https://doi.org/1 0.1109/LAWP.2016.2560904

17. Wu H et al (2020) A study on the fused deposition modeling process of graphene / Nano-Fe 304 composite absorber and its absorbing properties of electromagnetic microwave. Appl Sci 10(1508):1-12

18. Ren J, Yin JY (2018) 3D-printed low-cost dielectric-resonator-based ultrabroadband microwave absorber using carbon-loaded acrylonitrile butadiene styrene polymer. Materials (Basel) 11:1249. https://doi.org/10.3390/ma11 071249

19. Kjelgard KG, Wisland DT, Lande TS (2018) 3D printed wideband microwave absorbers using composite graphite / pla filament. In: Proceedings of 48th European Microwave Conference, pp 859-862

20. Khurram AA, Rakha SA, Ali N, Zhou P, Munir A (2014) Effect of low-content carbon nanotubes on the dielectric and microwave absorption properties of graphite / polymer nanocomposites. J Appl Polym Sci 40891(20):1-7. https://doi.org/10.1002/app.40891

21. Ansari A, Akhta MJ (2017) Co/graphite based light weight microwave absorber for electromagnetic shielding and stealth applications. Mater Res Express 4(1). https://doi.org/10.1088/2053-1591/aa570c

22. Arbaoui Y, Laur V, Maalouf A, Queffelec P, Passerieux D, Delias A, Blondy P (2016) Full 3-D printed microwave termination: a simple and low-cost solution. IEEE Trans Microw Theory Tech 64(1):271-278. https://doi.org/10.11 09/TMTT.2015.2504477

23. Xiong Y-J et al (2018) Structural broadband absorbing metamaterial based on three-dimensional printing technology. Acta Phys Sin 67(8):084202-1084202-8. https://doi.org/10.7498/aps.67.20172262

24. Hotta M, Hayashi M, Lanagan MT, Agrawal DK (2011) Complex permittivity of graphite, carbon black and coal powders in the ranges of X-band frequencies ( 8 2 to $12.4 \mathrm{GHz}$ ) and between 1 and $10 \mathrm{GHz}$. ISIJ Int 51(11):1766-1772

25. Kruth JP, Wang $X$, Laoui $T$, Froyen $L$ (2003) Lasers and materials in selective laser sintering. Assem Autom 23(4):357-371. https://doi.org/10.1108/014451 50310698652

26. J. L. Kenis, "ABS Technics," 2020. [Online]. Available: www.abstechnics.com.

27. IEEE (1998) IEEE Recommended Practice for Radio-Frequency ( RF ) Absorber Evaluation in the Range of $30 \mathrm{MHz}$ to $5 \mathrm{GHz}$; ANSI/IEEE Std. 11281998. IEEE, New York

28. Fan S, Song Y (2018) Bandwidth-enhanced polarization-insensitive metamaterial absorber based on fractal structures. J Appl Phys 123(8): 085110-1-085110-8. https://doi.org/10.1063/1.5004629

29. Beeharry T, Yahiaoui R, Selemani K, Ouslimani HH (2018) A co-polarization broadband radar absorber for RCS reduction. Materials (Basel) 11:1-11. https://doi.org/10.3390/ma11091668

30. Abdulkarim Yl, Deng L, Luo H, Huang S, Sabah C, Karaaslan M (2020) Electromagnetic simulations of polarization-insensitive and wide-angle multiband metamaterial absorber by incorporating double asterisk resonator. Bull Mater Sci 43(116):9. https://doi.org/10.1007/s12034-020-02098-3

31. R. K. Ganeriwala, "Multiphysics modelling of selective laser sintering/melting," PhD Thesis, University of California, 2015.

\section{Publisher's Note}

Springer Nature remains neutral with regard to jurisdictional claims in published maps and institutional affiliations. 